\title{
How Bright is the Proton? A Precise Determination of the Photon Parton Distribution Function
}

\author{
Aneesh Manohar, ${ }^{1,2}$ Paolo Nason, ${ }^{3}$ Gavin P. Salam, ${ }^{2, *}$ and Giulia Zanderighi ${ }^{2,4}$ \\ ${ }^{1}$ Department of Physics, University of California at San Diego, La Jolla, California 92093, USA \\ ${ }^{2}$ CERN, Theoretical Physics Department, CH-1211 Geneva 23, Switzerland \\ ${ }^{3}$ INFN, Sezione di Milano Bicocca, 20126 Milan, Italy \\ ${ }^{4}$ Rudolf Peierls Centre for Theoretical Physics, 1 Keble Road, University of Oxford, Oxford OXI 3NP, United Kingdom
}

(Received 29 July 2016; published 9 December 2016)

\begin{abstract}
It has become apparent in recent years that it is important, notably for a range of physics studies at the Large Hadron Collider, to have accurate knowledge on the distribution of photons in the proton. We show how the photon parton distribution function (PDF) can be determined in a model-independent manner, using electron-proton ( $e p$ ) scattering data, in effect viewing the $e p \rightarrow e+X$ process as an electron scattering off the photon field of the proton. To this end, we consider an imaginary, beyond the Standard Model process with a flavor changing photon-lepton vertex. We write its cross section in two ways: one in terms of proton structure functions, the other in terms of a photon distribution. Requiring their equivalence yields the photon distribution as an integral over proton structure functions. As a result of the good precision of $e p$ data, we constrain the photon PDF at the level of $1 \%-2 \%$ over a wide range of momentum fractions.
\end{abstract}

DOI: 10.1103/PhysRevLett.117.242002

A fast-moving particle generates an associated electromagnetic field which can be interpreted as a distribution of photons, as originally calculated by Fermi, Weizsäcker, and Williams [1-3] for pointlike charges. The corresponding determination of the photon distribution for hadrons, specifically $f_{\gamma / p}$ for the proton, has, however, been the subject of debate over recent years.

The photon distribution is small compared to that of the quarks and gluons, since it is suppressed by a power of the electromagnetic coupling $\alpha$. Nevertheless, it has been realized in the past few years that its poor knowledge is becoming a limiting factor in our ability to predict key scattering reactions at CERN's Large Hadron Collider (LHC). Notable examples are the production of the Higgs boson through $W / Z$ fusion [4], or in association with an outgoing weak boson [5]. For $W^{ \pm} H$ production it is the largest source of uncertainty [6]. The photon distribution is also potentially relevant for the production of lepton pairs [7-11], top quarks [12], pairs of weak bosons [13-18], and generally enters into electroweak corrections for almost any LHC process. The diphoton excess around $750 \mathrm{GeV}$ seen by ATLAS and CMS $[19,20]$ has also generated interest in understanding $f_{\gamma / p}$.

The two most widely used estimates of $f_{\gamma / p}$ are those included in the MRST2004QED [21] and NNPDF23QED [22] parametrizations of the proton structure. In the NNPDF

Published by the American Physical Society under the terms of the Creative Commons Attribution 3.0 License. Further distribution of this work must maintain attribution to the author(s) and the published article's title, journal citation, and DOI. approach, the photon distribution is constrained mainly by LHC data on the production of pairs of leptons, $p p \rightarrow \ell^{+} \ell^{-}$. This is dominated by $q \bar{q} \rightarrow \ell^{+} \ell^{-}$, with a small component from $\gamma \gamma \rightarrow \ell^{+} \ell^{-}$. The drawback of this approach is that even with very small uncertainties in $\ell^{+} \ell^{-}$ production data [8], in the QCD corrections to $q \bar{q} \rightarrow \ell^{+} \ell^{-}$ and in the quark and anti-quark distributions, it is difficult to obtain high-precision constraints on $f_{\gamma / p}$.

In the MRST2004QED approach, the photon is instead modeled. It is assumed to be generated as emissions from free, pointlike quarks, using quark distributions fitted from deep-inelastic scattering (DIS) and other data. The free parameter in the model is an effective mass scale below which quarks stop radiating, which was taken in the range between current-quark masses (a few $\mathrm{MeV}$ ) and constituent-quark masses (a few hundred MeV). A more sophisticated approach [23] supplements a model of the photon component generated from quarks ("inelastic" part) with a calculation of the "elastic" component (whose importance has been understood at least since the early 1970s [24]) generated by coherent radiation from the proton as a whole. This was recently revived in Refs. [25-27]. Such an approach was also adopted for the CT14QED_INC [28] set, which further constrains the effective mass scale in the inelastic component using $e p \rightarrow e \gamma+X$ data [29], sensitive to the photon in a limited momentum range through the reaction $e \gamma \rightarrow e \gamma$ [30].

In this Letter we point out that electron-proton $(e p)$ scattering data already contain all the information that is needed to accurately determine $f_{\gamma / p}$. It is common to think of $e p$ scattering as a process in which a photon emitted from the electron probes the structure of the proton. 
However, one can equivalently think of it as an electron probing the photon field generated by the proton itself. Thus the ep scattering cross section is necessarily connected with $f_{\gamma / p}$. (This point of view is implicit also in Refs. [31-33].) A simple way to make the connection manifest is to consider, instead of ep scattering, the fictitious process $l+p \rightarrow L+X$, where $l$ and $L$ are neutral leptons, with $l$ massless and $L$ massive with mass $M$. We assume a transition magnetic moment coupling of the form $\mathcal{L}_{\text {int }}=(e / \Lambda) \bar{L} \sigma^{\mu \nu} F_{\mu \nu} l$. Here, $e^{2}\left(\mu^{2}\right) /(4 \pi) \equiv \alpha\left(\mu^{2}\right)$ is the $\overline{\mathrm{MS}}$ QED coupling evaluated at the scale $\mu$, and the arbitrary scale $\Lambda \gg \sqrt{s}$ (where $\sqrt{s}$ is the center-of-mass energy) is introduced to ensure the correct dimensions.

The crucial observation that we rely on is inspired in part by Drees and Zeppenfeld's study of supersymmetric particle production at $e p$ colliders [34]: there are two ways of writing the heavy-lepton production cross section $\sigma$, one in terms of standard proton structure functions, $F_{2}$ and $F_{L}$ (or $F_{1}$ ), the other in terms of the proton PDFs $f_{a / p}$, where the dominant flavor that contributes will be $a=\gamma$. Equating the latter result with the former will allow us to determine $f_{\gamma / p}$.

We start with the inclusive cross section for $l(k)+p(p) \rightarrow$ $L\left(k^{\prime}\right)+X$. Defining $q=k-k^{\prime}, Q^{2}=-q^{2}$, and $x_{B \mathrm{j}}=$ $Q^{2} /(2 p q)$, we have

$$
\begin{aligned}
\sigma= & \frac{1}{4 p k} \int \frac{d^{4} q}{(2 \pi)^{4} q^{4}} e_{\mathrm{ph}}^{2}\left(q^{2}\right)\left[4 \pi W_{\mu \nu}(p, q) L^{\mu \nu}(k, q)\right] \\
& \times 2 \pi \delta\left((k-q)^{2}-M^{2}\right),
\end{aligned}
$$

where the proton hadronic tensor (as defined in Ref. [35]) is given by $W_{\mu \nu}(p, q)=-g_{\mu \nu} F_{1}\left(x_{B \mathrm{j}}, Q^{2}\right)+p_{\mu} p_{\nu} /$ $(p q) F_{2}\left(x_{B \mathrm{j}}, Q^{2}\right)$ up to terms proportional to $q_{\mu}, q_{\nu}$, and the leptonic tensor is $L^{\mu \nu}(k, q)=\frac{1}{2}\left[e_{\mathrm{ph}}^{2}\left(q^{2}\right) / \Lambda^{2}\right]$ $\times \operatorname{Tr}\left(k\left[\mathscr{q}, \gamma^{\mu}\right]\left(k^{\prime}+M\right)\left[\gamma^{\nu}, \mathscr{q}\right]\right)$. In Eq. (1) we introduced the physical QED coupling

$$
e_{\mathrm{ph}}^{2}\left(q^{2}\right)=e^{2}\left(\mu^{2}\right) /\left\{1-\Pi\left[q^{2}, \mu^{2}, e^{2}\left(\mu^{2}\right)\right]\right\},
$$

where $\Pi$ is the photon self-energy and $\mu$ is the renormalization scale. We stress that Eq. (1) is accurate up to corrections of order $\sqrt{s} / \Lambda$, since neither the electromagnetic current nor the $\bar{L} \gamma l$ vertex are renormalized.

We find

$$
\begin{aligned}
\sigma= & \frac{c_{0}}{2 \pi} \int_{x}^{1-\left(2 x m_{p} / M\right)} \frac{d z}{z} \int_{Q_{\min }^{2}}^{Q_{\max }^{2}} \frac{d Q^{2}}{Q^{2}} \alpha_{\mathrm{ph}}^{2}\left(-Q^{2}\right)\left[\left(2-2 z+z^{2}\right.\right. \\
& \left.+\frac{2 x^{2} m_{p}^{2}}{Q^{2}}+\frac{z^{2} Q^{2}}{M^{2}}-\frac{2 z Q^{2}}{M^{2}}-\frac{2 x^{2} Q^{2} m_{p}^{2}}{M^{4}}\right) F_{2}\left(x / z, Q^{2}\right) \\
& \left.+\left(-z^{2}-\frac{z^{2} Q^{2}}{2 M^{2}}+\frac{z^{2} Q^{4}}{2 M^{4}}\right) F_{L}\left(x / z, Q^{2}\right)\right],
\end{aligned}
$$

where $x=M^{2} /\left(s-m_{p}^{2}\right), \quad m_{p}$ is the proton mass, $F_{L}\left(x, Q^{2}\right)=\left(1+4 m_{p}^{2} x^{2} / Q^{2}\right) F_{2}\left(x, Q^{2}\right)-2 x F_{1}\left(x, Q^{2}\right)$ and $c_{0}=16 \pi^{2} / \Lambda^{2}$. Assuming that $M^{2} \gg m_{p}^{2}$, we have $Q_{\min }^{2}=x^{2} m_{p}^{2} /(1-z)$ and $Q_{\max }^{2}=M^{2}(1-z) / z$.

The same result in terms of parton distributions can be written as

$$
\sigma=c_{0} \sum_{a} \int_{x}^{1} \frac{d z}{z} \hat{\sigma}_{a}\left(z, \mu^{2}\right) \frac{M^{2}}{z s} f_{a / p}\left(\frac{M^{2}}{z s}, \mu^{2}\right),
$$

where, in the $\overline{\mathrm{MS}}$ factorization scheme,

$$
\begin{aligned}
\hat{\sigma}_{a}\left(z, \mu^{2}\right)= & \alpha\left(\mu^{2}\right) \delta(1-z) \delta_{a \gamma}+\frac{\alpha^{2}\left(\mu^{2}\right)}{2 \pi}[-2+3 z \\
& \left.+z p_{\gamma q}(z) \ln \frac{M^{2}(1-z)^{2}}{z \mu^{2}}\right] \sum_{i \in\{q, \bar{q}\}} e_{i}^{2} \delta_{a i}+\cdots,
\end{aligned}
$$

where $e_{i}$ is the charge of quark flavor $i$ and $z p_{\gamma q}(z)=1+(1-z)^{2}$. To understand which terms we choose to keep, observe that the photon will be suppressed by $\alpha L$ relative to the quark and gluon distributions, which are of order $\left(\alpha_{s} L\right)^{n}$, where $L=\ln \mu^{2} / m_{p}^{2} \sim 1 / \alpha_{s}$. The contribution proportional to $F_{2}$ in Eq. (3) is of order $\alpha^{2} L\left(\alpha_{s} L\right)^{n}$, while that proportional to $F_{L}$ is of order $\alpha^{2}\left(\alpha_{s} L\right)^{n}$. We neglect terms that would be of order $\alpha^{3} L\left(\alpha_{s} L\right)^{n}$ or $\alpha^{2} \alpha_{s}\left(\alpha_{s} L\right)^{n}$. By requiring the equivalence of Eqs. (3) and (4) up to the orders considered, one obtains (in the $\overline{\mathrm{MS}}$ scheme)

$$
\begin{aligned}
x f_{\gamma / p}\left(x, \mu^{2}\right)= & \frac{1}{2 \pi \alpha\left(\mu^{2}\right)} \int_{x}^{1} \frac{d z}{z}\left\{\int_{\left(x^{2} m_{p}^{2} / 1-z\right)}^{\left(\mu^{2} / 1-z\right)} \frac{d Q^{2}}{Q^{2}} \alpha^{2}\left(Q^{2}\right)\right. \\
& \times\left[\left(z p_{\gamma q}(z)+\frac{2 x^{2} m_{p}^{2}}{Q^{2}}\right) F_{2}\left(x / z, Q^{2}\right)\right. \\
& \left.-z^{2} F_{L}\left(\frac{x}{z}, Q^{2}\right)\right] \\
& \left.-\alpha^{2}\left(\mu^{2}\right) z^{2} F_{2}\left(\frac{x}{z}, \mu^{2}\right)\right\}
\end{aligned}
$$

where the result includes all terms of order $\alpha L\left(\alpha_{s} L\right)^{n}$, $\alpha\left(\alpha_{s} L\right)^{n}$, and $\alpha^{2} L^{2}\left(\alpha_{s} L\right)^{n}$ [36]. Within our accuracy $\alpha_{\mathrm{ph}}\left(-Q^{2}\right) \approx \alpha\left(Q^{2}\right)$. The conversion to the $\overline{\mathrm{MS}}$ factorization scheme, the last term in Eq. (6), is small (see Fig. 2).

From Eq. (6) we have derived expressions up to order $\alpha \alpha_{s}$ for the $P_{\gamma q}, P_{\gamma g}$, and $P_{\gamma \gamma}$ splitting functions using known results for the $F_{2}$ and $F_{L}$ coefficient functions and for the QED $\beta$ function. Those expressions agree with the results of a direct evaluation in Ref. [37].

The evaluation of Eq. (6) requires information on $F_{2}$ and $F_{L}$. First (and somewhat unusually in the context of modern PDF fits), we will need the elastic contributions to $F_{2}$ and $F_{L}$, 


$$
\begin{gathered}
F_{2}^{\mathrm{el}}\left(x, Q^{2}\right)=\frac{\left[G_{E}\left(Q^{2}\right)\right]^{2}+\left[G_{M}\left(Q^{2}\right)\right]^{2} \tau}{1+\tau} \delta(1-x), \\
F_{L}^{\mathrm{el}}\left(x, Q^{2}\right)=\frac{\left[G_{E}\left(Q^{2}\right)\right]^{2}}{\tau} \delta(1-x),
\end{gathered}
$$

where $\tau=Q^{2} /\left(4 m_{p}^{2}\right)$ and $G_{E}$ and $G_{M}$ are the electric and magnetic Sachs form factors of the proton [see, e.g., Eqs. (19) and (20) of Ref. [38]]. A widely used approximation for $G_{E, M}$ is the dipole form $G_{E}\left(Q^{2}\right)=1 /\left(1+Q^{2} / m_{\text {dip }}^{2}\right)^{2}, G_{M}\left(Q^{2}\right)=\mu_{p} G_{E}\left(Q^{2}\right)$, with $m_{\text {dip }}^{2}=0.71 \mathrm{GeV}^{2}$ and $\mu_{p} \simeq 2.793$. This form is of interest for understanding qualitative asymptotic behaviors, predicting $f_{\gamma / p}(x) \sim \alpha(1-x)^{4}$ at large $x$ dominated by the magnetic component, and $x f_{\gamma / p}(x) \sim \alpha \ln 1 / x$ at small $x$ dominated by the electric component. However, for accurate results, we will rather make use of a recent fit to precise world data by the A1 collaboration [39], which shows clear deviations from the dipole form, with an impact of up to $10 \%$ on the elastic part of $f_{\gamma / p}(x)$ for $x \lesssim 0.5$. The data constrain the form factors for $Q^{2} \lesssim 10 \mathrm{GeV}^{2}$. At large $x$, Eq. (6) receives contributions only from $Q^{2}>x^{2} m_{p}^{2} /(1-x)$, which implies that the elastic contribution to $f_{\gamma} / p$ is known for $x \lesssim 0.9$. Note that the last term in Eq. (6) does not have an elastic contribution for large $\mu^{2}$ because of the rapid dropoff of $G_{E, M}$.

The inelastic components of $F_{2}$ and $F_{L}$ contribute for $W^{2}=m_{p}^{2}+Q^{2}(1-x) / x>\left(m_{p}+m_{\pi^{0}}\right)^{2}$. One needs data over a large range of $x$ and $Q^{2}$. This is available thanks to a long history of $e p$ scattering studies. We break the inelastic part of the $\left(x, Q^{2}\right)$ plane into three regions, as illustrated in Fig. 1. In the resonance region, $W^{2} \lesssim 3.5 \mathrm{GeV}^{2}$ we use a fit to data by CLAS [40], and also consider an alternative fit to the world data by Christy and Bosted (CB) [41]. In the low$Q^{2}$ continuum region we use the GD11-P fit by HERMES [42] based on the ALLM parametric form [43]. Both the GD11-P and CB resonance fits are constrained by photoproduction data, i.e., they extend down to $Q^{2}=0$. The

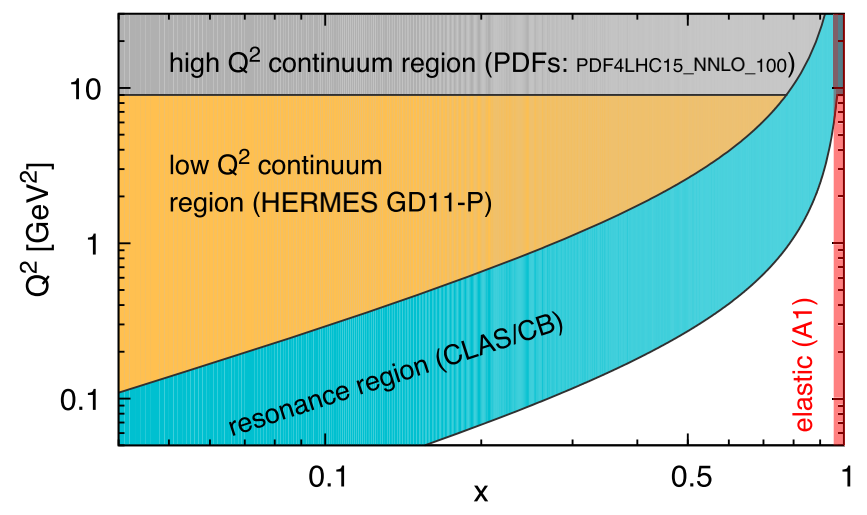

FIG. 1. Our breakup of the $\left(x, Q^{2}\right)$ plane and the data for $F_{2}\left(x, Q^{2}\right)$ and $F_{L}\left(x, Q^{2}\right)$ we use in each region. The white region is inaccessible at leading order in QED.
CLAS fit also behaves sensibly there. (Very low $Q^{2}$ values play little role because the analytic properties of the $W^{\mu \nu}$ tensor imply that $F_{2}$ vanishes as $Q^{2}$ at fixed $W^{2}$.) These fits are for $F_{2}\left(x, Q^{2}\right)$. We also require $F_{L}$, or, equivalently, $R=\sigma_{L} / \sigma_{T}$, which are related by

$F_{L}\left(x, Q^{2}\right)=F_{2}\left(x, Q^{2}\right)\left(1+\frac{4 m_{p}^{2} x^{2}}{Q^{2}}\right) \frac{R\left(x, Q^{2}\right)}{1+R\left(x, Q^{2}\right)}$,

and we use the parametrization for $R$ from HERMES [42], extended to vanish smoothly as $Q^{2} \rightarrow 0$. The leading twist contribution to $F_{L}$ is suppressed by $\alpha_{s}\left(Q^{2}\right) /(4 \pi)$. At high $Q^{2}$ we determine $F_{2}$ and $F_{L}$ from the PDF4LHC15_NNLO_100 [44] merger of next-to-next-toleading order (NNLO) [45,46] global PDF fits [47-49], using massless NNLO coefficient functions [50-53] implemented in HOPPET [54-56].

In Fig. 2 we show the various contributions to our photon PDF, which we dub "LUXQED," as a function of $x$, for a representative scale choice of $\mu=100 \mathrm{GeV}$. There is a sizable elastic contribution, with an important magnetic component at large values of $x$. The white line represents contributions arising from the $Q^{2}<1$ region of all the structure functions, including the full elastic contribution. For the accuracy we are aiming at, all contributions that we have considered, shown in Fig. 2, have to be included, and inelastic contributions with $Q^{2}<1$ cannot be neglected. The photon momentum fraction is $0.43 \%$ at $\mu=100 \mathrm{GeV}$.

In Fig. 3 we show the sources contributing to the uncertainty on our calculation of $f_{\gamma / p}$ at our reference scale $\mu=100 \mathrm{GeV}$. They are stacked linearly and consist of a conservative estimate of $\pm 50 \%$ for the uncertainty on $R=\sigma_{L} / \sigma_{T}$ at scales $Q^{2}<9 \mathrm{GeV}^{2}(\mathrm{R})$; standard 68\% C.L. uncertainties on the PDFs, applied to scales $Q^{2} \geq 9 \mathrm{GeV}^{2}$ (PDF); a conservative estimate of the uncertainty on the

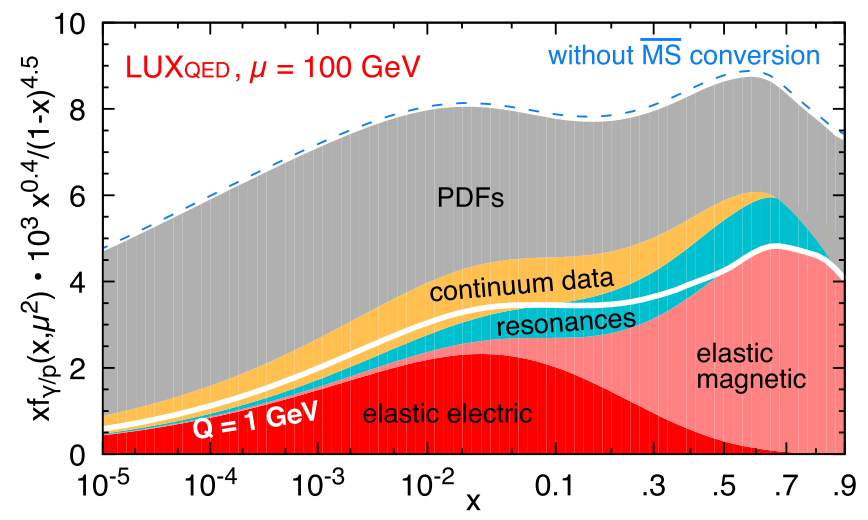

FIG. 2. Contributions to the photon PDF at $\mu=100 \mathrm{GeV}$, multiplied by $10^{3} x^{0.4} /(1-x)^{4.5}$, from the various components discussed in the text. For the inelastic part, the area below the white line is the contribution from $Q^{2} \leq 1(\mathrm{GeV})^{2}$ in Eq. (6) including the elastic part. The PDF would be the dashed blue line without the $\overline{\mathrm{MS}}$ conversion term. 


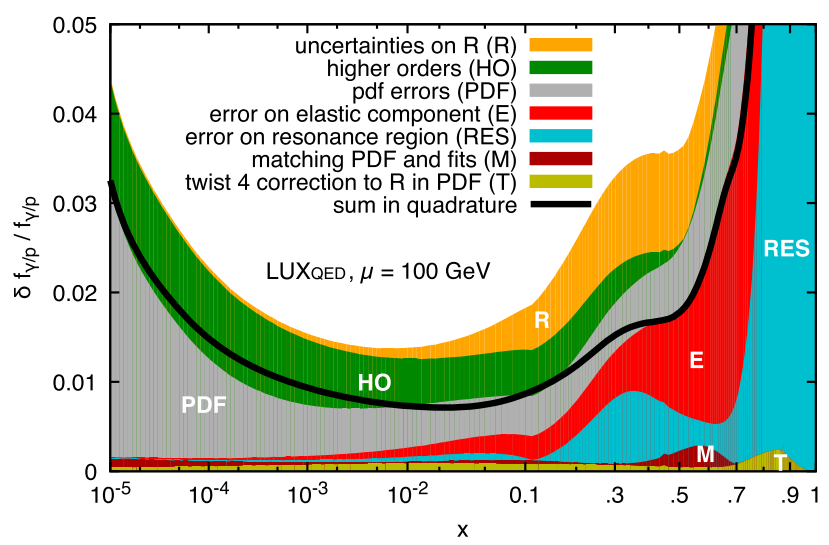

FIG. 3. Linearly stacked relative uncertainties on the photon PDF, from all sources we have considered, and their total sum in quadrature shown as a black line, which is our final uncertainty.

elastic form factors, equal to the sum in quadrature of the fit error and of the estimated size of the two-photon exchange contribution in Ref. [39] (E); an estimate of the uncertainty in the resonance region taken as the difference between the CLAS and CB fits (RES); a systematic uncertainty due to the choice of the transition scale between the HERMES $F_{2}$ fit and the perturbative determination from the PDFs, obtained by reducing the transition scale from 9 to $5 \mathrm{GeV}^{2}(\mathrm{M})$; missing higher order effects, estimated using a modification of Eq. (6), with the upper bound of the $Q^{2}$ integration set to $\mu^{2}$ and the last term adjusted to maintain $\alpha^{2}\left(\alpha_{s} L\right)^{n}$ accuracy (HO); a potential twist- 4 contribution to $F_{L}$ parametrized as a factor $\left(1+5.5 \mathrm{GeV}^{2} / Q^{2}\right)$ [57] for $Q^{2} \geq 9 \mathrm{GeV}^{2}(\mathrm{~T})$. One-sided errors are all symmetrized. Our final uncertainty, shown as a solid line in Fig. 3, is obtained by combining all sources in quadrature and is about $1 \%-2 \%$ over a large range of $x$ values.

In Fig. 4 we compare our LUXQED result for the $\overline{\mathrm{MS}} f_{\gamma / p}$ to determinations available publicly within LHAPDF [58]. Of the model-based estimates, CT14QED_INC [28] and MRST2004 [21], CT14QED_INC is in good agreement with LUXQED within its uncertainties. Its model for the inelastic component is constrained by $e p \rightarrow e \gamma+X$ data from ZEUS [29] and includes an elastic component. Note, however, that for the neutron CT14QED_INC neglects the important neutron magnetic form factor. As for the modelindependent determinations, NNPDF30[59], which notably extends NNPDF23[22] with full treatment of $\alpha\left(\alpha_{s} L\right)^{n}$ terms in the evolution [60], almost agrees with our result at small $x$. At large $x$ its band overlaps with our result, but the central value and error are both much larger.

Similar features are visible in the corresponding $\gamma \gamma$ partonic luminosities, defined as

$$
\frac{d L_{\gamma \gamma}}{d \ln M^{2}}=\frac{M^{2}}{s} \int \frac{d z}{z} f_{\gamma / p}\left(z, M^{2}\right) f_{\gamma / p}\left(\frac{M^{2}}{z s}, M^{2}\right),
$$

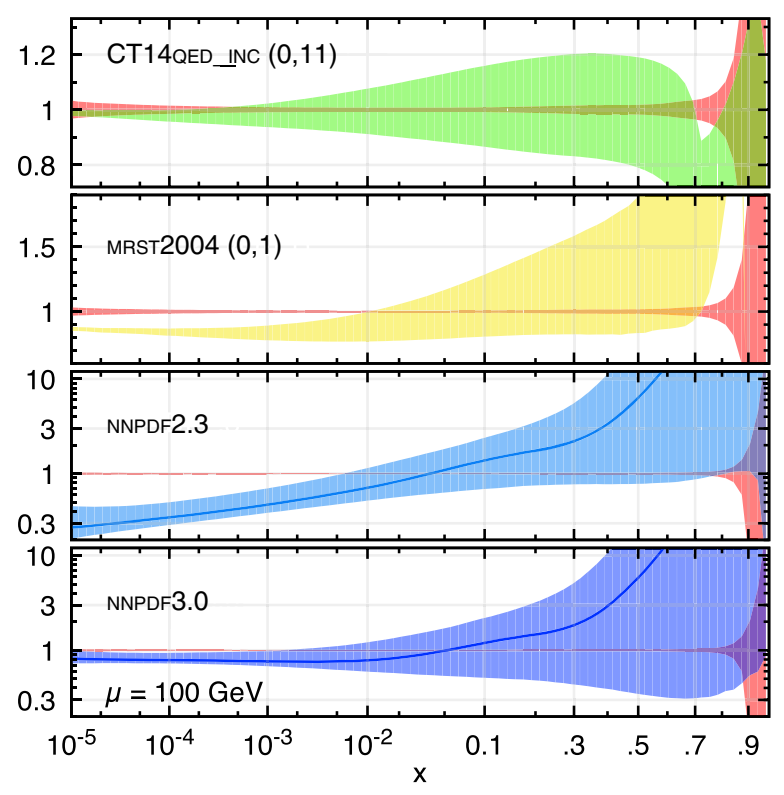

FIG. 4. The ratio of common PDF sets to our LUXQED result, along with the LUXQED uncertainty band (light red). The CT14 and MRST bands correspond to the range from the PDF members shown in brackets $(68 \% \mathrm{cl}$. in CT14's case). The NNPDF bands span from $\max \left(\mu_{r}-\sigma_{r}, r_{16}\right)$ to $\mu_{r}+\sigma_{r}$, where $\mu_{r}$ is the average (represented by the blue line), $\sigma_{r}$ is the standard deviation over replicas, and $r_{16}$ denotes the $16^{\text {th }}$ percentile among replicas. Note the different $y$ axes for the panels.

and shown in Fig. 5, as a function of the $\gamma \gamma$ invariant mass $M$, for several center-of-mass energies.

As an application, we consider $p p \rightarrow H W^{+}\left(\rightarrow \ell^{+} \nu\right)+X$ at $\sqrt{s}=13 \mathrm{TeV}$, for which the total cross section without photon-induced contributions is $91.2 \pm 1.8 \mathrm{fb}$ [6], with the error dominated by (nonphotonic) PDF uncertainties. Using HAWK2.0.1[61], we find a photon-induced contribution of $5.5_{-2.9}^{+4.3} \mathrm{fb}$ with NNPDF30, to be compared to $4.4 \pm 0.1 \mathrm{fb}$ with LUXQED.

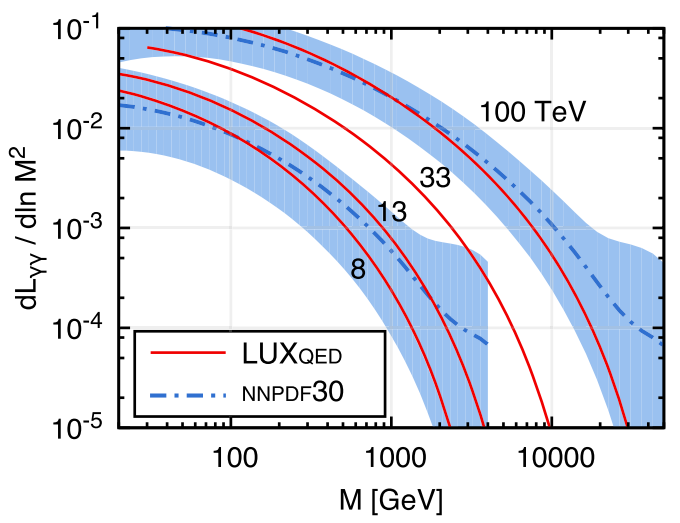

FIG. 5. $\gamma \gamma$ luminosity in $p p$ collisions as a function of the $\gamma \gamma$ invariant mass $M$, at four collider center-of-mass energies. The NNPDF30 results are shown only for 8 and $100 \mathrm{TeV}$. The uncertainty of our LUXQED results is smaller than the width of the lines. 
In conclusion, we have obtained a formula [i.e., Eq. (6)] for the $\overline{\mathrm{MS}}$ photon PDF in terms of the proton structure functions, which includes all terms of order $\alpha L\left(\alpha_{s} L\right)^{n}, \alpha\left(\alpha_{s} L\right)^{n}$, and $\alpha^{2} L^{2}\left(\alpha_{s} L\right)^{n}$. Our method can be easily generalized to higher orders in $\alpha_{s}$ and holds for any hadronic bound state. Using current experimental information on $F_{2}$ and $F_{L}$ for protons we obtain a photon PDF with much smaller uncertainties than existing determinations, as can be seen from Fig. 4. The photon PDF has a substantial contribution from the elastic form factor $(\sim 20 \%)$ and from the resonance region $(\sim 5 \%)$, even for high values of $\mu \sim 100-1000 \mathrm{GeV}$. Our photon distribution, incorporating quarks and gluons from PDF4LHC15_NNLO_100 [44] and evolved with a QED-extended version of HOPPET is available as part of the LHAPDF library as the PDF4LHC15_NNLO_100 set and can also be obtained from [62]. More details of our analysis, including a derivation using PDF operators, computation of splitting functions, higher order corrections to Eq. (6), as well as an extension to the polarized case will be given in a longer publication [63].

We would like to thank Silvano Simula who provided us with a code for the CLAS parametrization, Jan Bernauer for discussions of the A1 results and fits, and Gunar Schnell for bringing the HERMES GD11-P fit to our attention and providing the corresponding code. We also thank Markus Diehl, Stefan Dittmaier, Stefano Forte, Kirill Melnikov, and Jesse Thaler for helpful discussions. This work was supported in part by ERC Consolidator Grant HICCUP (No. 614577), ERC Advanced Grant Higgs@LHC (No. 321133), a grant from the Simons Foundation (No. 340281 to A. M.), by DOE Grant No. DE-SC0009919, and NSF Grant No. NSF PHY11-25915. We also acknowledge MITP (G. Z.) and KITP (G. P. S., G. Z.) for hospitality while this work was being completed.

* On leave from CNRS, UMR 7589, LPTHE, F-75005, Paris, France.

[1] E. Fermi, Z. Phys. 29, 315 (1924).

[2] C. F. von Weizsacker, Z. Phys. 88, 612 (1934).

[3] E. J. Williams, Phys. Rev. 45, 729 (1934).

[4] M. Ciccolini, A. Denner, and S. Dittmaier, Phys. Rev. D 77, 013002 (2008).

[5] A. Denner, S. Dittmaier, S. Kallweit, and A. Muck, J. High Energy Phys. 03 (2012) 075.

[6] D. de Florian et al. (LHC Higgs Cross Section Working Group Collaboration), arXiv:1610.07922.

[7] G. Aad et al. (ATLAS Collaboration), Eur. Phys. J. C 76, 291 (2016).

[8] G. Aad et al. (ATLAS Collaboration), J. High Energy Phys. 08 (2016) 009.

[9] E. Accomando, J. Fiaschi, F. Hautmann, S. Moretti, and C. H. Shepherd-Themistocleous, arXiv:1606.06646 [Phys. Rev. D (to be published)].

[10] S. Alioli et al., arXiv:1606.02330.

[11] D. Bourilkov, arXiv:1606.00523.
[12] D. Pagani, I. Tsinikos, and M. Zaro, Eur. Phys. J. C 76, 479 (2016).

[13] M. Luszczak, A. Szczurek, and C. Royon, J. High Energy Phys. 02 (2015) 098.

[14] A. Denner, S. Dittmaier, M. Hecht, and C. Pasold, J. High Energy Phys. 02 (2016) 057.

[15] M. Ababekri, S. Dulat, J. Isaacson, C. Schmidt, and C. P. Yuan, arXiv:1603.04874.

[16] B. Biedermann, A. Denner, S. Dittmaier, L. Hofer, and B. Jäger, Phys. Rev. Lett. 116, 161803 (2016).

[17] B. Biedermann, M. Billoni, A. Denner, S. Dittmaier, L. Hofer, B. Jäger, and L. Salfelder, J. High Energy Phys. 06 (2016) 065.

[18] W. Yong, Z. Ren-You, M. Wen-Gan, L. Xiao-Zhou, and G. Lei, Phys. Rev. D 94, 013011 (2016).

[19] Tech. Rep. ATLAS-CONF-2015-081, CERN, Geneva (2015), http://cds.cern.ch/record/2114853.

[20] Tech. Rep. CMS-PAS-EXO-15-004, CERN, Geneva (2015), https://cds.cern.ch/record/2114808.

[21] A. D. Martin, R. G. Roberts, W. J. Stirling, and R. S. Thorne, Eur. Phys. J. C 39, 155 (2005).

[22] R. D. Ball, V. Bertone, S. Carrazza, L. Del Debbio, S. Forte, A. Guffanti, N. P. Hartland, and J. Rojo (NNPDF Collaboration), Nucl. Phys. B877, 290 (2013).

[23] M. Gluck, C. Pisano, and E. Reya, Phys. Lett. B 540, 75 (2002).

[24] V. M. Budnev, I. F. Ginzburg, G. V. Meledin, and V. G. Serbo, Phys. Rep. 15, 181 (1975).

[25] A. D. Martin and M. G. Ryskin, Eur. Phys. J. C 74, 3040 (2014).

[26] L. A. Harland-Lang, V. A. Khoze, and M. G. Ryskin, Eur. Phys. J. C 76, 255 (2016).

[27] L. A. Harland-Lang, V. A. Khoze, and M. G. Ryskin, J. High Energy Phys. 03 (2016) 182.

[28] C. Schmidt, J. Pumplin, D. Stump, and C. P. Yuan, Phys. Rev. D 93, 114015 (2016).

[29] S. Chekanov et al. (ZEUS Collaboration), Phys. Lett. B 687, 16 (2010).

[30] A. De Rujula and W. Vogelsang, Phys. Lett. B 451, 437 (1999).

[31] H. Anlauf, H. D. Dahmen, P. Manakos, T. Mannel, and T. Ohl, Comput. Phys. Commun. 70, 97 (1992).

[32] A. Mukherjee and C. Pisano, Eur. Phys. J. C30, 477 (2003).

[33] M. Łuszczak, W. Schäfer, and A. Szczurek, Phys. Rev. D 93, 074018 (2016).

[34] M. Drees and D. Zeppenfeld, Phys. Rev. D 39, 2536 (1989).

[35] K. A. Olive et al. (Particle Data Group), Chin. Phys. C 38, 090001 (2014).

[36] The part of the integrand in square brackets appears also in Refs. [31,32], though the integration limits differ. Ref. [33] also obtained a result in terms of structure functions, however its Eq. (3.25) is incompatible with leading order QED DGLAP evolution.

[37] D. de Florian, G. F. R. Sborlini, and G. Rodrigo, Eur. Phys. J. C 76, 282 (2016).

[38] G. Ricco, S. Simula, and M. Battaglieri, Nucl. Phys. B555, 306 (1999).

[39] J. C. Bernauer et al. (A1 Collaboration), Phys. Rev. C 90, 015206 (2014). 
[40] M. Osipenko et al. (CLAS Collaboration), Phys. Rev. D 67, 092001 (2003).

[41] M. E. Christy and P. E. Bosted, Phys. Rev. C 81, 055213 (2010).

[42] A. Airapetian et al. (HERMES Collaboration), J. High Energy Phys. 05 (2011) 126.

[43] H. Abramowicz, E. M. Levin, A. Levy, and U. Maor, Phys. Lett. B 269, 465 (1991).

[44] J. Butterworth et al., J. Phys. G 43, 023001 (2016).

[45] S. Moch, J. A. M. Vermaseren, and A. Vogt, Nucl. Phys. B688, 101 (2004).

[46] A. Vogt, S. Moch, and J. A. M. Vermaseren, Nucl. Phys. B691, 129 (2004).

[47] R. D. Ball et al. (NNPDF Collaboration), J. High Energy Phys. 04 (2015) 040.

[48] L. A. Harland-Lang, A. D. Martin, P. Motylinski, and R. S. Thorne, Eur. Phys. J. C 75, 204 (2015).

[49] S. Dulat, T.-J. Hou, J. Gao, M. Guzzi, J. Huston, P. Nadolsky, J. Pumplin, C. Schmidt, D. Stump, and C. P. Yuan, Phys. Rev. D 93, 033006 (2016).

[50] W. L. van Neerven and E. B. Zijlstra, Phys. Lett. B 272, 127 (1991).

[51] E. B. Zijlstra and W. L. van Neerven, Nucl. Phys. B383, 525 (1992).

[52] W. L. van Neerven and A. Vogt, Nucl. Phys. B568, 263 (2000).
[53] W. L. van Neerven and A. Vogt, Nucl. Phys. B588, 345 (2000).

[54] G. P. Salam and J. Rojo, Comput. Phys. Commun. 180, 120 (2009).

[55] M. Cacciari, F. A. Dreyer, A. Karlberg, G. P. Salam, and G. Zanderighi, Phys. Rev. Lett. 115, 082002 (2015).

[56] F. A. Dreyer and A. Karlberg, Phys. Rev. Lett. 117, 072001 (2016).

[57] A. M. Cooper-Sarkar, I. Abt, B. Foster, M. Wing, V. Myronenko, and K. Wichmann, Proceedings of the 24th International Workshop on Deep-Inelastic Scattering and Related Subjects (DIS 2016) Hamburg, Germany, April 1115, 2016, http://inspirehep.net/record/1466126/files/arXiv: 1605.08577.pdf.

[58] A. Buckley, J. Ferrando, S. Lloyd, K. Nordström, B. Page, M. Rüfenacht, M. Schönherr, and G. Watt, Eur. Phys. J. C 75, 132 (2015).

[59] V. Bertone and S. Carrazza, arXiv:1606.07130.

[60] V. Bertone, S. Carrazza, and J. Rojo, Comput. Phys. Commun. 185, 1647 (2014).

[61] A. Denner, S. Dittmaier, S. Kallweit, and A. Mück, Comput. Phys. Commun. 195, 161 (2015).

[62] http://cern.ch/luxqed.

[63] A. Manohar, P. Nason, G. P. Salam, and G. Zanderighi, in preparation. 